\title{
MECANISMOS DE AJUSTE ANTE RESTRICCIONES EN EL USO DEL AGUA SUBTERRÁNEA EN MÉXICO: ANÁLISIS DE ESCENARIOS CON UN MODELO ECONÓMICO INTERREGIONAL
}

\author{
Ph.D. López-Morales, Carlos ${ }^{1}$
}

De acuerdo con la Comisión Nacional del Agua mexicana (CNA), 100 de los 653 acuíferos de México se encuentran sobreexplotados. Un diagnóstico aquí reportado documenta que 64 de estos acuíferos pertenecen a las cinco regiones con baja disponibilidad de agua, juntas responsables del $50 \%$ del producto interno bruto nacional (PIB), mientras que los 36 restantes se ubican en cuatro regiones con disponibilidad media, donde se genera el 36\% del PIB. El objetivo de este trabajo es aplicar un modelo económico de comercio interregional basado en la ventaja comparativa para estudiar mecanismos de ajuste ante un escenario que limita la extracción subterránea al volumen de recarga anual. Los resultados informan que la sustitución de agricultura de temporal por irrigación es un mecanismo de ajuste dominante, mientras que la sustitución de extracciones superficiales por subterráneas, si bien se observa, es menos importante. El uso más intensivo de tierra de temporal, en general menos eficiente que la irrigada, se traduce en una elevación de los precios de los alimentos de 11\% respecto a la situación base. Estos hallazgos sugieren la importancia de asumir una visión integral en el manejo de los recursos naturales, pues la restricción en uno de ellos (agua subterránea) puede elevar las presiones sobre otros (tierra agrícola de temporal), como en el experimento aquí reportado.

Palabras clave: Agua subterránea, agua superficial, México, modelo interregional de insumoproducto, agricultura, programación lineal.
According to Mexico's National Water Commission, one in six Mexican aquifers is in overdraft. A diagnosis presented in this paper shows that $64 \%$ of these aquifers are located in the five regions of low water availability, together responsible for $50 \%$ of the national gross domestic product (GDP), while the remaining 36\% are located in four regions of medium water availability, where $36 \%$ of Mexico's GDP is generated. The aim of this paper is to apply an inter-regional economic trade model based on the theory of comparative advantage to study mechanisms of adjustment in a scenario that constrains groundwater use to annual recharge rates. Results obtained with the model suggest that there is a dominant adjustment process in which non-irrigated agriculture substitutes for irrigated agriculture. Another adjustment mechanism observed, although less intensively, is one in which surface withdrawals substitute for groundwater withdrawals. The more intensive use of non-irrigated land, generally less efficient relative to irrigated land, translates into an $11 \%$ rise in food prices relative to the baseline situation. These findings highlight the importance of an integrated vision of natural resources management, for the restriction on uses of one particular resource (groundwater) can raise the pressure on other resources (nonirrigated agricultural land).

JEL classification: Q56, Q57, Q58, Q25 Keywords: Groundwater, surface water, Mexico, inter-regional input-output model, agriculture, linear programming.

${ }^{1}$ Investigador postdoctoral. Instituto Global para la Sostenibilidad. Instituto Tecnológico de Estudios Superiores 


\section{Introducción}

La potencial severidad de una crisis de agua a escala global ha puesto al recurso hídrico en la agenda principal de los funcionarios y de los investigadores de diversas disciplinas. La todavía creciente población global y sus patrones de consumo cada vez más complejos implican una demanda global por agua que presiona una oferta que no se distribuye homogéneamente en el espacio ni en el tiempo, e intensifica la competencia entre usos alternativos elevando la frecuencia con la que el agua se convierte en factor limitante de la actividad económica. Por lo anterior, no es sorpresa que exista un llamado global a reconocer y comprender la dimensión económica del agua (ver, por ejemplo, ICWE, 1992).

Tal dimensión tiene dos aspectos principales. El agua es tanto un bien de consumo final para los hogares, como un factor de la producción esencial para actividades agrícolas e industriales. A pesar de la atención mayoritaria al primer aspecto, el uso doméstico del agua llega a solo el 11\% de los 3800 km3 de la extracción global anual (FAO, 2012); la agricultura tiene el uso más frecuente, con el $70 \%$ de dicho volumen; mientras que la industria, principalmente la generación de energía en termoeléctricas, utiliza lo restante (FAO, 2012). Esta distribución indica que el agua es más frecuentemente un factor de la producción que un bien de consumo final.

Los factores de la producción tradicionales en economía han sido capital, trabajo y ocasionalmente un concepto muy amplio de tierra. Sin embargo, las presiones sobre el medio ambiente han reclamado en décadas recientes una representación más elaborada de los recursos naturales como factores de la producción con el objetivo de entender cómo las economías hacen uso de ellos.
La caracterización tecnológica de un sistema económico, por ejemplo a través de una descripción de la red de transacciones intersectoriales e interregionales, ayuda a cuantificar en escenarios de escasez la tasa con la que el sistema económico demanda insumos producidos y no producidos en un período determinado.

Para el caso del agua, a dicha descripción hay que añadir estudios sobre la disponibilidad existente, aunque la definición de las dotaciones de agua económicamente relevantes es asunto complejo que requiere el establecimiento de puentes multidisciplinarios con la hidrología y con la geografía, principalmente. La complejidad del agua como factor económico en parte se basa porque tiene características tanto de un recurso renovable como de uno no renovable. Por un lado, la precipitación anual asociada al ciclo hidrológico puede tener magnitud y temporalidad relativamente estable, lo que hace del agua un recurso renovable cuyo uso estará finalmente limitado por el flujo de precipitación anual (Gleick, 2009; Ehrlich y Holdren, 1977). Sin embargo, el escurrimiento anual y la recarga de acuíferos derivados de la precipitación no constituyen medidas realistas de la disponibilidad económica del recurso, puesto que la infraestructura de extracción, ya sea superficial o subterránea, tiene capacidades limitadas (Postel, 1996).

Por otro lado, muchos de los acervos subterráneos de agua dulce exhiben una tasa de regeneración que puede ser muy baja, incluso cero, por lo que pueden ser descritos como no renovables. En tales situaciones, el uso del agua se encuentra finalmente limitada por los acervos existentes o por la tasas de recarga natural (Gleick, 2009). El volumen de agua potencialmente disponible en cualquier parte del mundo, entonces, es una combinación de los límites impuestos 
por acervos no renovables, por la magnitud de los flujos renovables y por la capacidad de extracción de la infraestructura existente.

El objetivo del presente trabajo es estudiar los mecanismos económicos de ajuste ante escenarios de escasez de agua a través de la aplicación, para el caso de México, de un modelo económico de comercio interregional basado en la teoría de la ventaja comparativa que somete la dependencia del sistema económico a la disponibilidad regional de los recursos naturales. El modelo aquí presentado está basado en Duchin (2005), quien formula el caso general, y en López-Morales y Duchin (2011) quienes lo aplican para el análisis del uso y disponibilidad de agua en la economía mexicana. Este trabajo contribuye con la distinción entre agua superficial y agua subterránea, omitida en los estudios referidos, y simula un escenario en el que las extracciones de agua subterránea se limitan a los flujos renovables de recarga de acuíferos. Se estudian dos mecanismos económicos de ajuste, que tienen que ver con la sustitución de agricultura de irrigación por agricultura de temporal, o de secano, que no presiona sobre los acervos de agua líquida o azul, y con la sustitución de extracciones de fuentes subterráneas por extracciones de fuentes superficiales.

El documento está organizado de la siguiente manera: sigue a esta sección introductoria una breve descripción de la situación del agua en México; la sección 3 presenta los antecedentes en la literatura atinentes a las herramientas particulares aquí presentadas; la sección 4 presenta los métodos analíticos y la estrategia de implementación por escenarios; la sección 5 presenta algunos resultados; y la sección 6 concluye.

\section{Agua en México}

Debido a que el agua se distribuye en el territorio de una forma desigual, la situación en México se expone mejor usando las 13 regiones hidrológicoadministrativas en las que la Comisión Nacional del Agua (CNA) mexicana divide al territorio (figura 1). Para obtener las fronteras de estas unidades territoriales, la CNA agrega los límites municipales para hacer coincidir, tanto como sea posible, los límites de la división política con los límites de 37 regiones hidrológicas.

Figura 1. Regiones hidrológicoadministrativas de México

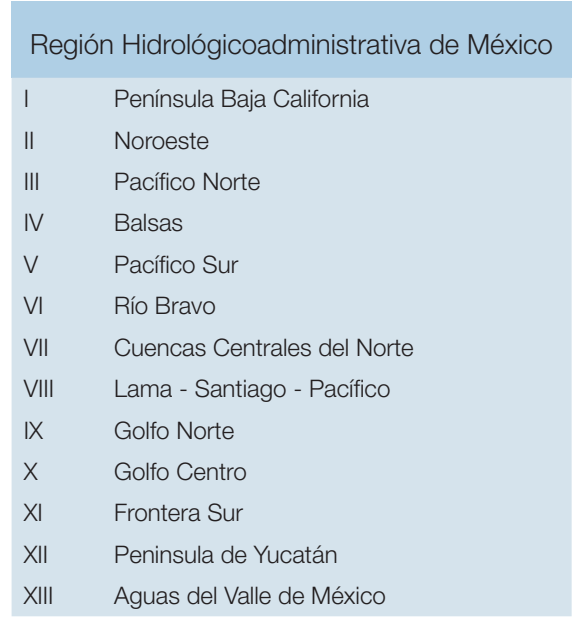

Fuente: CNA (2010).

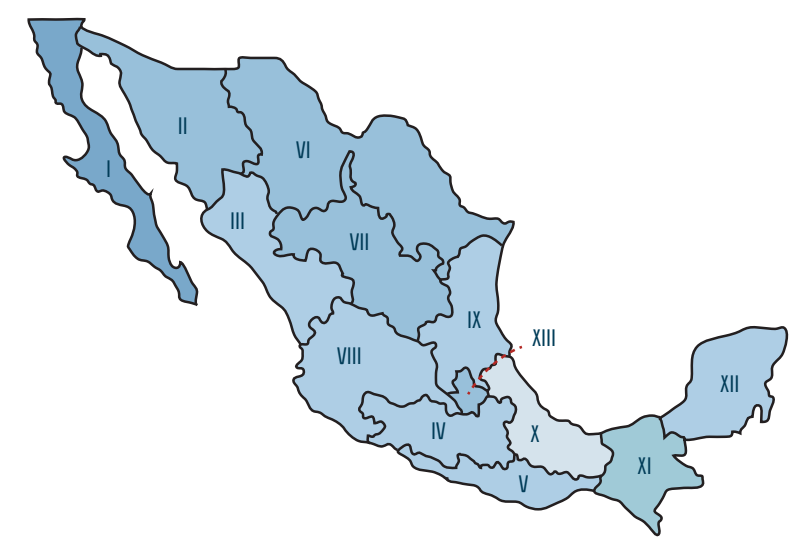


La distribución del agua renovable, o la suma del escurrimiento superficial y la recarga de acuíferos (FAO, 2012), en estas trece regiones se expone en la tabla 1. La distribución geográfica permite crear tres grupos de dis- ponibilidad (alta, media y baja) con fines de exposición. Dicho ordenamiento, además, sigue un patrón en el que la disponibilidad de agua crece conforme las regiones se van ubicando al sur del territorio.

Tabla 1. Distribución regional del agua renovable

\begin{tabular}{lcc} 
Región & $\begin{array}{c}\text { Agua renovable } \\
\text { Km } 3 / \text { año }\end{array}$ & $\%$ \\
II & 4.62 & 1.0 \\
II & 8.2 & 1.8 \\
VI & 12.02 & 2.6 \\
VII & 7.78 & 1.7 \\
XIII & 3.51 & 0.8 \\
III & 25.63 & 5.6 \\
IV & 21.66 & 4.7 \\
VIII & 34.04 & 7.4 \\
IX & 25.5 & 5.6 \\
V & 32.79 & 7.1 \\
XII & 29.65 & 6.5 \\
X & 95.46 & 20.8 \\
XI & 157.75 & 34.4 \\
Nacional & 458.61 & 100.0 \\
\hline
\end{tabular}

Fuente: CNA (2010).

La figura 2 compara la distribución regional de agua renovable con las distribuciones regionales de la población y de la actividad económica. Las regiones de baja disponibi- lidad de agua, con 9\% del agua renovable total, albergan a $40 \%$ de la población y generan 51\% del producto interno bruto nacional; mientras que las de disponibilidad media,

Figura 2. Distribución regional del agua renovable, del producto interno bruto y de la población

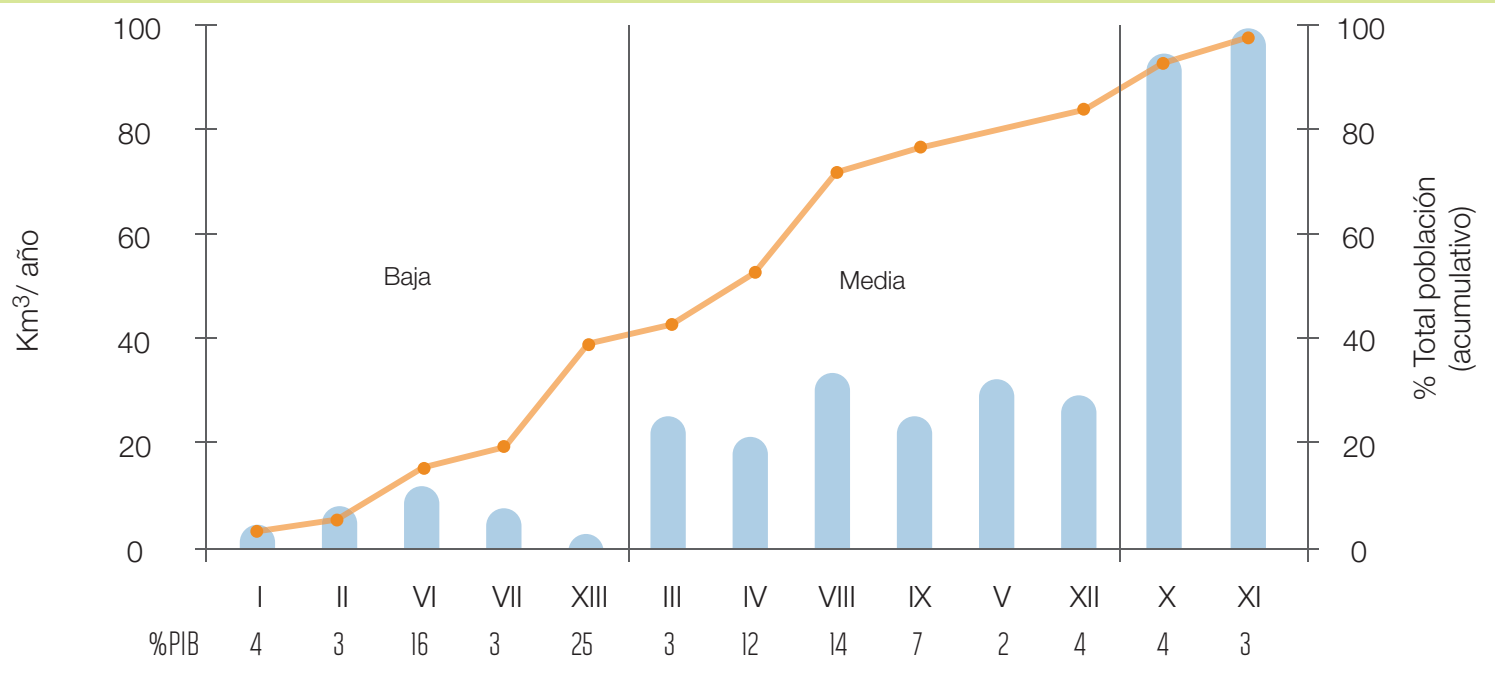

Nota. Las barras representan el agua renovable regional (escala izquierda). La línea muestra la distribución acumulada de la población en cada región (escala derecha). Los números debajo de los numerales romanos de las regiones indican la participación de cada región en el producto interno bruto. Las regiones están ordenadas por grupos de disponibilidad (Ver Tabla 1). Fuente: Elaboración propia con datos de CNA (2010). 
con $37 \%$ del agua renovable, albergan a un $50 \%$ de la población y generan el $42 \%$ del producto interno bruto; y las regiones de disponibilidad alta, con 55\% del agua renovable nacional, albergan al $10 \%$ de la población y generan el $7 \%$ del producto interno bruto nacional.

La figura 3 muestra las extracciones de agua por región y por sector de actividad económica. El mayor volumen de extracciones se realiza en las regiones de disponibilidad baja y media, donde se concentran población y actividad económica. En el nivel nacional, alrededor del $65 \%$ de las extracciones se hace para riego agrícola, que también es el uso mayoritario en todas las regiones excepto aquellas con grandes centros urbanos (como la XIII, que alberga a la zona metropolitana de la Ciudad de México). El servicio público, que es una categoría que abastece a la industria y a los servicios en centros urbanos, consume el $13 \%$ de las extracciones nacionales de agua; por su parte, la industria autoabastecida utiliza el 17\%; la generación de electricidad (en termoeléctricas) lo hace con poco menos del $5 \%$; y el servicio doméstico utiliza el restante. La distribución sectorial de los usos del agua permite ver que en México el agua es más frecuentemente un factor de la producción que un bien de consumo final.

Figura 3. México 2004: Extracciones de agua por sector de actividad y región hidrológica

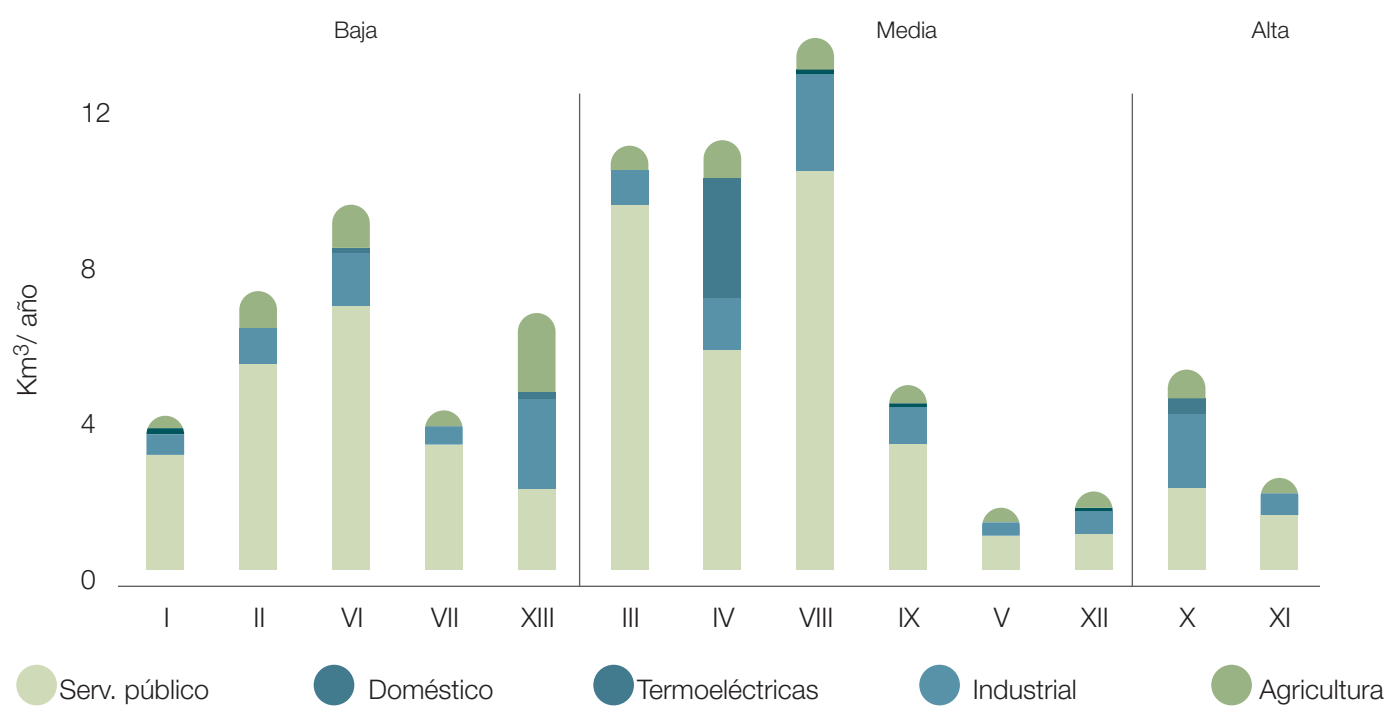

Fuente: Elaboración propia con datos de CNA (2010).

Es importante notar que estas figuras se ción, los retornos al sistema hidrológico son refieren a las concesiones de agua por uso de relevancia. El escurrimiento del agua de económico tal y como están reportadas en riego de los campos agrícolas, por ejemplo, el Registro Público de Derechos de Agua de la CNA (CNA, 2010). Son una medida de aproximación a los retiros que la actividad económica hace de fuentes superficiales o subterráneas. Ocurre con el agua que, a diferencia de otros factores de la producpuede aumentar la disponibilidad para usos cuenca abajo, mientras que el agua utilizada para procesos de enfriamiento industrial se suele regresar a la fuente de donde ha sido extraída. El dilema entre contabilizar extracciones o contabilizar el consumo neto de 
retornos ha recibido atención en la literatura que busca entender la economía del agua (ver, para un tratamiento inicial, Seckler, 1996). Actualmente, el estado del arte se centra en la relación de estas últimas con la actividad económica regional y agregada, al no existir una contabilización de los usos consuntivos y los retornos con el detalle existente para los datos sobre extracciones.

Finalmente, la tabla 2 muestra para todas las regiones la partición del agua renovable entre superficial y subterránea, el volumen de extracciones por fuente y la distribución de acuíferos por región hidrológicoadministrativa. El $80 \%$ del agua renovable en México proviene de fuentes superficiales y el $20 \%$ restante en subterráneas, en la recarga de acuíferos; el $60 \%$ de las extracciones nacionales son superficiales y el $40 \%$ restante son de fuentes subterráneas. De acuerdo con la CNA, el 15\% de los 653 acuíferos del país se encuentran sobreexplotados y se concentran en las cinco regiones del grupo de baja disponibilidad y en cuatro regiones del grupo de disponibilidad media.

En particular, para las regiones Noroeste (II), Río Bravo (VI), Cuencas Centrales del Norte (VII), Lerma-Santiago-Pacífico (VIII) y Aguas del Valle de México (XIII) el porcentaje de acuíferos sobreexplotados con relación al total regional es superior al porcentaje nacional. 64 de los acuíferos sobreexplotados se concentran en el grupo de baja disponibilidad, donde se genera el $51 \%$ del PIB nacional (figura 2), mientras que los 36 restantes se ubican en cuatro regiones del grupo de disponibilidad media, donde se genera el $36 \%$ del PIB nacional. En suma, las regiones que albergan a los acuíferos sobreexplotados son responsables del $87 \%$ del PIB de México, y albergan a cerca del $75 \%$ de la población nacional.

Tabla 2. Distribución regional del agua renovable y de las extracciones por tipo de fuente

\begin{tabular}{|c|c|c|c|c|c|c|c|c|}
\hline \multirow[b]{2}{*}{$\begin{array}{l}\text { Disponibilidad } \\
\text { de agua }\end{array}$} & \multirow[b]{2}{*}{ Región } & \multicolumn{2}{|c|}{ Agua Renovable } & \multicolumn{2}{|c|}{ Extracciones } & \multicolumn{2}{|c|}{ Número de acuiferos } & \multirow{2}{*}{$\begin{array}{l}\% \text { acuiferos } \\
\text { sobre } \\
\text { explotados } \\
\text { por región }\end{array}$} \\
\hline & & \multicolumn{2}{|c|}{$\mathrm{Km}^{3} /$ año } & \multicolumn{2}{|c|}{$\begin{array}{c}\text { Superficial Subterránea } \\
\mathrm{Km}^{3} \text { / año }\end{array}$} & \multirow{2}{*}{$\begin{array}{c}\text { Total } \\
87\end{array}$} & \multirow{2}{*}{$\begin{array}{c}\begin{array}{c}\text { Sobre } \\
\text { explotados }\end{array} \\
8\end{array}$} & \\
\hline Ваја & 1 & 3.37 & 1.25 & 1.99 & 2.06 & & & 9 \\
\hline & $\|$ & 5.07 & 3.13 & 4.07 & 2.77 & 63 & 13 & 21 \\
\hline & $\mathrm{VI}$ & 6.86 & 5.17 & 4.64 & 4.56 & 100 & 15 & 15 \\
\hline & VII & 5.51 & 2.27 & 1.24 & 2.73 & 68 & 24 & 35 \\
\hline & XIII & 1.68 & 1.83 & 2.59 & 3.18 & 14 & 4 & 29 \\
\hline \multirow[t]{6}{*}{ Media } & III & 22.36 & 3.26 & 9.6 & 1.49 & 24 & 2 & 8 \\
\hline & IV & 17.06 & 4.6 & 9.04 & 2.11 & 46 & 2 & 4 \\
\hline & VIII & 26.35 & 7.69 & 7.54 & 7.02 & 127 & 30 & 24 \\
\hline & $\mathrm{IX}$ & 24.23 & 1.27 & 3.79 & 1.09 & 40 & 2 & 5 \\
\hline & V & 30.8 & 1.99 & 0.96 & 0.42 & 35 & 0 & 0 \\
\hline & XII & 4.33 & 25.32 & 0.03 & 1.92 & 4 & 0 & 0 \\
\hline \multirow[t]{3}{*}{ Alta } & $x$ & 91.61 & 3.85 & 5.13 & 0.97 & 22 & 0 & 0 \\
\hline & XI & 139.74 & 18.02 & 1.61 & 0.65 & 23 & 0 & 0 \\
\hline & Vacional & 378.97 & 79.65 & 52.23 & 30.97 & 653 & 100 & 15 \\
\hline
\end{tabular}

\section{Estudios previos}

Existen diversos estudios que tienen motivaciones similares a las de este documento. La literatura relevante incluye aplicaciones de la economía de insumo-producto para el caso del agua, y aplicaciones de modelos de equilibrio general computable (EGC). Las aplicaciones de la economía de insumo-producto se dividen, a su vez, en dos pequeños grupos. El primero de ellos se conforma con estudios que cuantifican el uso directo e indi- 
recto del agua por los sectores económicos, como son los casos de Duarte et al. (2002), Velázquez (2006) y Llop (2008) para el caso de España y de algunas de sus regiones; de Guan y Hubacek (2008) y Hubacek y Sun (2005), para el caso de China, aunque ellos también se interesan en estimar los requerimientos hidrológicos de disolución de contaminantes, en el primer caso, y los efectos de un cambio de dietas y de tecnologías en China, en el segundo; y de Lenzen y Foran (2001) y de Lenzen (2009) para Australia.

El segundo grupo de estudios con economía insumo-producto se conforma por estudios que utilizan técnicas de optimización y por tanto están más cercanos a la metodología aquí presentada. En dos aportaciones tempranas, Henry y Bowen (1981) y Harris y Rea (1984) desarrollan modelos similares de programación lineal para realizar una valoración económica de las dotaciones de agua para dos economías regionales de Estados Unidos. Su metodología consiste en estimar los precios sombra asociados al volumen de los acervos físicos, en el primer caso: y al volumen del flujo anual superficial, en el segundo. También con programación lineal, Liu et al. (2009) obtienen los precios sombra del agua para nueve regiones hidrológicas de China. He et al. (2006) y He et al. (2007) hacen lo propio pero con un método un tanto distinto: un modelo dinámico y lineal de equilibrio general computable.

A diferencia de los estudios arriba referidos, las aplicaciones de modelos en la tradición del equilibrio general computable se han hecho mayoritariamente en una escala global. Calzadilla et al. (2008), por ejemplo, extienden la base de datos del Proyecto de Análisis del Comercio Mundial (GTAP, por sus siglas en inglés) para estudiar los efectos globales de una mejora generalizada en las técnicas de irrigación. Berritella et al. (2007) estudian los impactos de varios escenarios de disponibilidad de agua subterránea en diversas partes del mundo; y Berrittella et al. (2005) analizan el impacto en la economía mundial del establecimiento de políticas de precios de agua en diferentes regiones del mundo.

El presente trabajo se distingue de los dos grupos de estudios ya referidos en lo siguiente. A diferencia de los estudios de insumo-producto, este estudio toma en cuenta las dotaciones de agua regionales de manera explícita. A diferencia de los estudios de programación lineal que también toman en cuenta dichas dotaciones, en este estudio se consideran las dotaciones de otros recursos naturales (i.e. tierra agrícola) y de otros factores de la producción (trabajo y capital). Finalmente, a diferencia de los estudios de EGC, que utilizan el supuesto de Armington para determinar flujos regionales de comercio, el modelo utilizado aquí tiene un mecanismo explícito de asignación de producto que se basa en la teoría de la ventaja comparativa.

\subsection{Antecedentes del modelo de comercio} interregional

El modelo utilizado en el presente estudio es una adaptación, para un solo país, del modelo de comercio mundial (WTM, su acrónimo en inglés) propuesto por Duchin (2005). El WTM es un modelo multirregional, multisectorial y multifactorial que toma características relevantes de la economía para estudiar las asignaciones de producto y los patrones de comercio determinados por la teoría de la ventaja comparativa. El modelo está diseñado expresamente para el análisis de comportamientos económicos del pasado o de diversos escenarios sobre las posibilidades presentes y futuras del desarrollo sustentable (Duchin, 2005). 
El modelo es un programa lineal que en su formulación original minimiza el uso de los factores de la producción sujeto a i) la generación de producto suficiente para satisfacer una demanda final determinada, ii) factibilidad en términos del uso y disponibilidad de los recursos productivos primarios, y iii) la ocurrencia de ganancias de comercio. La literatura tiene diversas extensiones y aplicaciones: Strömman y Duchin (2006) expanden el modelo original para incluir costos de transporte entre los socios comerciales; Juliá y Duchin (2007) estudian las capacidades del comercio de alimentos para adaptarse a los impactos del cambio climático; He y Duchin (2009) evalúan escenarios sobre desarrollo de infraestructura para promover el desarroIlo regional en China; y Strömman et al. (2009) resuelven el modelo en un marco multiobjetivo minimizando alternadamente uso de recursos y emisiones de CO2 para estudiar cómo puede el comercio internacional reducir las emisiones de dicho gas.

De modo similar a He y Duchin (2009), el modelo para este trabajo se aplicará para un análisis interregional de una economía nacional, aunque no se tomaran en cuenta los costos de transporte como en su caso, por lo que se deja para después el análisis de los flujos bilaterales de comercio entre regiones. De modo similar a Juliá y Duchin (2007), el modelo permite la elección de tecnologías alternativas para el sector agrícola (irrigación o no irrigación) en cada región. El enfoque particular en dicho tratamiento está basado, a su vez, en Duchin y Lange (1992). En Duchin y Levine (2011), se presenta una generalización de la elección de tecnologías para todos los sectores; y en Duchin y López-Morales (2012), se ejemplifican sus ventajas en el contexto de un modelo global de comercio. El modelo de este estudio se basa de modo más particular en las aplicaciones de López-Morales y Duchin (2011) para 13 regiones hidrológicoadministrativas de México, y constituye una extensión de dichas aplicaciones que distingue fuentes subterráneas y superficiales en la construcción de las dotaciones regionales de agua.

\section{Métodos}

4.1 Formulación del modelo El modelo es un programa líneal que minimiza el uso de los factores para satisfacer una demanda final dada sujeta a la disponibilidad regional de los factores productivos. El modelo incluye 13 regiones hidroeconómicas, 15 sectores económicos, listados en la tabla 3, y seis factores de la producción (dos clases de tierra: irrigada y de temporal, dos fuentes de agua: superficial y subterránea, trabajo y capital). El modelo está finalmente basado en la economía de insumo-producto, por lo que el proceso de generación de producto está descrito por conjuntos de coeficientes sectoriales específicos que muestran los requerimientos unitarios de los insumos producidos y de los no producidos. Para cada sector de actividad y región, este conjunto describe la tecnología particular que está en uso. La agrupación de dichos coeficientes técnicos para todos los sectores en matrices de coeficientes, Am para los insumos producidos y Fm para los no producidos en la región $m$, representa la estructura tecnológica agregada de la economía. Cualquier solución factible del modelo determina de modo implicito un patrón de comercio entre las regiones que, dado el objetivo de minimización de costos, seguirá el principio de ventaja comparativa. 
Tabla 3. Sectores de actividad y asignación de estados a regiones hidroeconómicas

\begin{tabular}{|c|c|c|c|c|}
\hline & \multirow[b]{2}{*}{ Sectores de actividad } & \multicolumn{3}{|c|}{ Regiones hidroeconómicas } \\
\hline & & $\begin{array}{l}\text { Disponibilidad } \\
\text { de agua }\end{array}$ & Estados & Región \\
\hline 1 & Agricultura & Baja & Baja California & I \\
\hline 2 & Ganadería, silvicultura y pesca & & Baja California Norte & \\
\hline 3 & Petróleo y gas & & Sonora & $\|$ \\
\hline 4 & Minería no petrolera & & Coahuila, Chihuahua & $\mathrm{Vl}$ \\
\hline 5 & Electricidad & & Nuevo León & \\
\hline 6 & Construcción & & Durango, Zacatecas & VII \\
\hline 7 & Procesamiento de alimentos & & San Luis Potosí & \\
\hline 8 & Manufacturas ligeras & & Estado de México & XIII \\
\hline 9 & Plásticos y químicos & & Distrito Federal & \\
\hline 10 & Manufacturas pesadas & Media & Sinaloa & III \\
\hline 11 & Comercio & & Guerrero, Michoacán & IV \\
\hline 12 & Transporte & & Puebla, Tlaxcala, Morelos & \\
\hline 13 & Servicios no-financieros & & Aguascalientes, Colima & VIII \\
\hline 14 & Servicios financieros & & Guanajuato, Jalisco, Nayarit & \\
\hline \multirow[t]{5}{*}{15} & Otros servicios & & $\begin{array}{l}\text { Hidalgo, Querétaro } \\
\text { Tamaulipas }\end{array}$ & IX \\
\hline & & & Oaxaca & V \\
\hline & & & $\begin{array}{c}\text { Campeche, Yucatán } \\
\text { Quintana Roo }\end{array}$ & XII \\
\hline & & Alta & Veracrúz & $x$ \\
\hline & & & Chiapas, Tabasco & $X I$ \\
\hline
\end{tabular}

Cada región $m$ tiene dos alternativas tecnológicas para generar producto agrícola, irrigación o temporal, y dos fuentes alternativas de agua, superficial o subterránea, determinando tres opciones tecnológicas para el sector agrícola (irrigación superficial, irrigación subterránea, o agricultura de temporal) y dos opciones para el resto de los sectores (extrayendo agua de fuentes superficiales o de fuentes subterráneas). El modelo contempla por tanto, incluyendo las 13 regiones subnacionales, 39 opciones para generar producto agrícola y 26 opciones para generar producto no agrícola.

Las opciones tecnológicas para los sectores agrícolas y no agrícolas son no excluyentes, en el sentido de que más de una opción puede estar activa en cada grupo de sectores. Por ejemplo, la agricultura de temporal, o de secano, puede coexistir en alguna región con la de irrigación con agua superficial, con agua subterránea, o con ambas. Con fines de implementación y de exposición, estas opciones tecnológicas se agrupan en el modelo en cuatro categorías / no excluyente, expuestas en la figura 4.

En la opción 1, los sectores no agrícolas extraen agua superficial y el sector agrícola depende del agua de lluvia. En la opción 2 los no agrícolas extraen agua superficial y el agrícola irriga con agua superficial. En la opción 3 los no agrícolas extraen agua subterránea y la agricultura es de temporal, mientras que en la opción 4 los sectores no agrícolas extraen agua subterránea y el agrícola irriga con agua subterránea. Estas opciones están disponibles para cada región y pueden estar activas con diferentes intensidades al mismo tiempo. Por último, cada una de estas opciones / se enfrenta a diferentes condiciones regionales de disponibilidad de los recursos no producidos de los que depende. 
Figura 4. Opciones tecnológicas regionales de acuerdo a acervo de agua

Fuente: Elaboración propia.

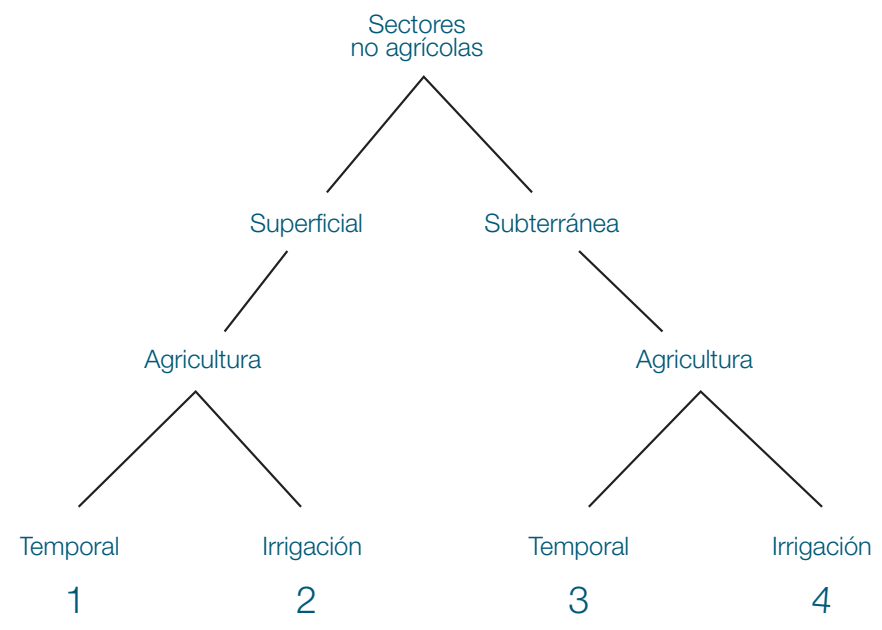

El conjunto de parámetros y de variables exógenas que el modelo requiere como datos consiste en i) matrices de coeficientes técnicos unitarios de uso de insumos producidos, $A_{m}$, y no producidos, $F_{m}$, ii) los componentes exógenos de los precios de los insumos no producidos, $\pi_{m}$, iii) las dotaciones regionales de insumos no producidos, $f_{m}$ y iv) la magnitud de la demanda final nacional a satisfacer, y. La tabla 4 expone la notación y las fuentes estadísticas para los parámetros y las variables exógenas. Para atender la ausencia de matrices regionales de coeficientes técnicos,

$A_{m}$, la implementación del modelo asume que la matriz nacional es representativa para todas las regiones. Este proceder permite la implementación del modelo aunque al costo de generalizar detalles regionales. Las matrices de requerimientos de bienes no producidos, $\mathbf{F}_{m}$, se construyen a partir de datos de uso de tierra, agua, mano de obra y capital, con gran detalle regional, por lo que estas matrices compensan la pérdida de información asociada por operar con una matriz A nacional.

Tabla 4. Notación y bases de datos para los parámetros y las variables del modelo

\begin{tabular}{|c|c|c|c|}
\hline & Notación & Descripción & Fuente \\
\hline Parámetros & $\begin{array}{l}A_{m} \\
F_{m}\end{array}$ & $\begin{array}{l}\text { Matríi insumo-producto } \\
\text { Matrices de insumos no producidos }\end{array}$ & $\begin{array}{c}\text { INEGI (2012a) } \\
\text { Tierra: SAGARPA (2011) } \\
\text { Trabajo y capital: INEGI (2012) } \\
\text { Agua: CNA (2010) }\end{array}$ \\
\hline \multirow[t]{2}{*}{ Variables exógenas } & $\mathrm{fm}_{\mathrm{m}}$ & Vectores de dotaciones de recursos & $\begin{array}{l}\text { Tierra: SAGARPA (2010), } \\
\text { SEMARNAT (2010) } \\
\text { Trabajo y capital: INEGI (2012a) } \\
\text { Agua: CNA (2010), este estudio }\end{array}$ \\
\hline & y & $\begin{array}{l}\text { Vector de demanda final } \\
\text { Vectores de precios de los insumos }\end{array}$ & $\begin{array}{c}\text { INEGI (2004) } \\
\text { Tierra: SAGARPA (2011), } \\
\text { Lee Harris (2002) } \\
\text { Trabajo y capital: INEGI (2012a), } \\
\text { INEGI (2012), } \\
\text { Blancas (2006), } \\
\text { Lee Haris (2002), } \\
\text { Agua: CNA (2010) }\end{array}$ \\
\hline Variables endógenas & $\begin{array}{c}x_{m} \\
p \\
r_{m}\end{array}$ & $\begin{array}{l}\text { Vectores de producto regional } \\
\text { Vector de precios nacionalel } \\
\text { Vectores de rentas por escasezez }\end{array}$ & Este estudio \\
\hline
\end{tabular}


Por ser un programa lineal, el modelo consta de una formulación primal y de una dual íntimamente asociadas. El modelo primal elige los niveles de actividad para cada una de las opciones tecnológicas disponibles para cada sector económico en cada región, por lo que resuelve un problema de asignación. El primal tiene el objetivo de minimizar el costo de uso de los insumos no producidos sujeto a su disponibilidad regional y la satisfacción de la demana final nacional. El modelo dual, en cambio, elige el nivel de precios de los bienes producidos, agrupados en el vector nacional p, y las rentas por escasez (o también, precios sombra) de los factores no producidos, agrupadas en vectores regionales $r_{m}$, por tanto resuelve un problema de valuación. El dual tiene el objetivo de maximizar el valor del producto final neto del valor en precios sombra de los recursos escasos, sujeto a que el precio unitario de cada bien producido debe cubrir el costo total de uso de los insumos totales requeridos en su producción.

Formalmente, los modelos son:

Modelo primal

$$
\operatorname{Min} \mathrm{Z}_{\mathrm{P}}=\sum_{m=1}^{13} \sum_{l=1}^{4} \boldsymbol{\pi}_{\mathbf{m}}^{\mathbf{m}} \mathbf{F}_{\mathbf{m}}^{\mathbf{l}} \mathbf{x}_{\mathbf{m}}^{\mathbf{1}}
$$

Sujeto a :

$$
\begin{aligned}
& \sum_{m=1}^{13} \sum_{l=1}^{4}\left(\mathbf{I}-\mathbf{A}_{\mathbf{m}}^{\mathbf{l}}\right) \mathbf{x}_{\mathbf{m}}^{\mathbf{l}} \geq \mathbf{y} \\
& \sum_{l=1}^{4} \mathbf{F}_{\mathbf{m}}^{\mathbf{l}} \mathbf{x}_{\mathbf{m}}^{\mathbf{l}} \leq \mathbf{f}_{\mathbf{m}} ; \forall m \\
& \mathbf{x}_{\mathbf{m}}^{\mathbf{l}} \geq \mathbf{0} ; \forall m, \forall l
\end{aligned}
$$

Modelo dual

$$
\operatorname{Max} \mathrm{Z}_{\mathrm{D}}=\mathbf{p}^{\prime} \mathbf{y}-\sum_{\mathbf{m}=\mathbf{1}}^{13} \mathbf{r}_{\mathbf{m}}^{\prime} \mathbf{f}_{\mathbf{m}}
$$

Sujeto a :

$$
\begin{aligned}
& \left(\mathbf{I}-\mathbf{A}_{\mathrm{m}}^{\mathrm{l}}\right) \mathbf{p}-\mathbf{F}_{\mathrm{m}}^{\mathrm{\prime l}} \mathbf{r}_{\mathrm{m}} \leq \mathbf{F}_{\mathrm{m}}^{\mathrm{r}} \boldsymbol{\pi}_{\mathrm{m}} ; \forall l, \forall m \\
& \mathbf{p} \geq \mathbf{0} ; \mathbf{r}_{\mathrm{m}} \geq \mathbf{0}, \forall m
\end{aligned}
$$

Dadas las propiedades de complementariedad del programa lineal, los vectores de variables de elección del modelo dual, p y los vectores $r_{m}$, están relacionados con las restricciones del modelo primal. Si las restricciones del primal

$$
\sum_{m=1}^{13} \sum_{l=1}^{4}\left(\mathbf{I}-\mathbf{A}_{\mathbf{m}}^{\mathbf{l}}\right) \mathbf{x}_{\mathbf{m}}^{\mathbf{l}} \geq \mathbf{y}
$$

se cumplen con igualdad, entonces $\mathbf{p}$ será estrictamente positivo (excepto en el caso de un programa lineal degenerado, en el que algún precio puede ser cero). De igual forma, si las restricciones del primal

$$
\sum_{l=1}^{4} \mathbf{F}_{\mathbf{m}}^{\mathbf{l}} \mathbf{x}_{\mathbf{m}}^{\mathbf{l}} \leq \mathbf{f}_{\mathbf{m}}
$$

se cumplen con igualdad, las rentas de escasez en $r_{m}$ serán estrictamente positivas (excepto en el caso degenerado, en el que alguna renta por escasez puede ser cero). Las variables $\mathbf{p}$ indican los precios de los bienes producidos cuando los insumos no producidos se valúan por su valor de mercado, $\boldsymbol{\pi}_{\mathrm{m}}$, y por las rentas de escasez $\mathbf{r}_{\mathrm{m}}$. En la medida en que los precios observados no incluyan a estas últimas, no corresponderán necesariamente con los valores de p obtenidos en el modelo. Por último, las restricciones duales

$$
\left(\mathbf{I}-\mathbf{A}_{\mathrm{m}}^{\mathrm{\prime l}}\right) \mathbf{p}-\mathbf{F}_{\mathrm{m}}^{\mathrm{\prime l}} \mathbf{r}_{\mathrm{m}} \leq \mathbf{F}_{\mathrm{m}}^{\mathrm{r}^{\mathrm{r}}} \boldsymbol{\pi}_{\mathrm{m}}
$$

relacionan las magnitudes entre estas variables.

Del teorema de dualidad en programación lineal se tiene que $Z P=Z D$ en la solución óptima, asegurando entonces que el valor de la demanda final de los bienes producidos, p'y, es equivalente al ingreso de los poseedores de los factores productivos a precios de mercado,

$$
\sum_{m=1}^{13} \sum_{l=1}^{4} \boldsymbol{\pi}_{\mathbf{m}}^{\prime} \mathbf{F}_{\mathbf{m}}^{\mathbf{l}} \mathbf{x}_{\mathbf{m}}^{\mathbf{l}}
$$

más la valoración a precios sombra de los insumos escasos,

$$
\sum_{m=1}^{13} \sum_{l=1}^{4} \boldsymbol{\pi}_{\mathbf{m}}^{\prime} \mathbf{F}_{\mathbf{m}}^{\mathbf{l}} \mathbf{x}_{\mathbf{m}}^{\mathbf{l}}
$$


Dado que el vector de precios $\mathbf{p}$ responde ante la emergencia de rentas de escasez para recursos que no necesariamente tienen un mercado establecido (por ejemplo, el agua), no puede ser interpretado como un vector de precios de mercado de los bienes finales. Para que p pueda ser interpretado de esa forma se requieren de supuestos adicionales sobre arreglos institucionales que indiquen la apropiación de las rentas de escases por los poseedores de los factores de la producción, cuestión que escapa al alcance de esta implementación.

\subsection{Diseño de escenarios} Dada la disponibilidad de la matriz de insumoproducto, el modelo se implementa y se calibra con datos del año 2004 y se diseña un escenario base para servir como referencia con el escenario que limita la extracción de agua subterránea a las tasas de recarga. El escenario base, por tanto, resulta de resolver el modelo ante la ausencia de restricciones a las extracciones de agua subterránea que luego se implementan en el segundo escenario, y es una representación estilizada de la economía que busca aproximar los comportamientos observados que son de interés.

Las dotaciones de tierra irrigada y no irrigada, de trabajo y de capital se determinan por las magnitudes observadas en las bases de datos listadas en la tabla 4, y se mantienen inalteradas en ambos escenarios. Para el caso del agua, la CNA mexicana ofrece datos de la oferta renovable de agua por fuente, mostrados para cada región en la tabla 2. Esta magnitud, sin embargo, no puede tomarse como dotación económicamente relevante debido a que tácitamente se asumiría una capacidad de extracción de la infraestructura existente tal que resulta irreal para algunas regiones, sobre todo aquellas ricas en agua.
El escenario base requiere, entonces, de una estimación de los recursos de agua explotables. Dicha estimación no está disponible en las bases de datos estudiadas. Por tal motivo, el escenario base toma como referencia el volumen de agua concesionado por el organismo operador como una aproximación y asume que existen porcentajes de capacidad ociosa equivalentes al 10\% para la extracción subterránea y al $30 \%$ para la extracción superficial. Si bien la elección de estos porcentajes es arbitraria, pretende representar el diferencial entre el caudal de diseño, o de capacidad máxima, de la infraestructura existente y el caudal que efectivamente esta tiliza.

El segundo escenario simula el establecimiento de restricciones a la extracción de agua subterránea limitándola para cada región a la magnitud de la recarga anual estimada por la CNA, y mantiene las dotaciones de agua superficial, asi como las de los otros recursos, equivalentes a las del escenario base. Es decir, que las extracciones de agua subterránea en cada región no podrán superar la magnitud de la tasa a la que los acervos subterráneos se renuevan. El análisis de escenarios estudia la respuesta del sistema económico enfocándose en dos opciones de ajuste. Primero, sustituciones de agricultura de irrigación por agricultura de temporal, que no compite por el agua azul. Segundo, sustitución de extracciones subterráneas con extracciones superficiales, en la medida en que estas sean factibles.

\section{Resultados}

\subsection{Escenario base}

La tabla 5 muestra la comparación del escenario base con los datos observados para el producto agrícola y para las extracciones de agua de ambas fuentes. El total nacional del producto agrícola es el mismo: el modelo 
solo modifica su distribución regional. Los totales por tipo de agricultura informan que el modelo predice mayor intensidad de la agricultura de irrigación, sobre todo en las regiones con baja y media disponibilidad de agua. En consecuencia, el modelo predice menos agricultura de temporal en el total nacional, y no predice agricultura de irrigación en las regiones $\mathrm{V}$, con disponibilidad media, $\mathrm{XyXI}$, con disponibilidad alta, al ser estas las que exhiben el mayor costo unitario de producción agrícola (ver apartado siguiente).

El escenario base reproduce el patrón de extracciones de agua observado en el nivel nacional, así como su distribución regional. Debido a que el modelo predice mayor irrigación que en los datos observados, el

\section{Tabla 5. Escenario base y datos observados: distribución regional del producto agrícola} por tipo de tecnología y de las extracciones de agua por tipo de fuente

\begin{tabular}{|c|c|c|c|c|c|c|c|c|c|c|c|c|c|}
\hline \multirow{3}{*}{$\begin{array}{l}\text { Disponibilidac } \\
\text { de agua }\end{array}$} & \multirow{3}{*}{ Región } & \multicolumn{6}{|c|}{ Producto agrícola (mmdp) } & \multicolumn{6}{|c|}{ Extracciones de agua ( $\left.\mathrm{Km}^{3} / \mathrm{año}\right)$} \\
\hline & & \multicolumn{3}{|c|}{ Observado } & \multicolumn{3}{|c|}{ Modelo } & \multicolumn{3}{|c|}{ Observadas } & \multicolumn{3}{|c|}{ Modelo } \\
\hline & & Tempora & al Irrigación & Total & Temporal & Irrigación & Total & Superficial & Subterráne & eaTotals & Superficial & Subterráne & eaTotal \\
\hline \multirow[t]{5}{*}{ Baja } & I & 0.11 & 8.43 & 8.54 & 0.14 & 9.69 & 9.83 & 1.99 & 2.06 & 4.05 & 2.11 & 2.37 & 4.48 \\
\hline & $\|$ & 0.14 & 12.13 & 12.27 & 0.19 & 13.95 & 14.14 & 4.07 & 2.77 & 6.84 & 4.64 & 3.18 & 7.82 \\
\hline & $\mathrm{Vl}$ & 2.38 & 13.67 & 16.05 & 3.10 & 15.72 & 18.82 & 4.64 & 4.56 & 9.20 & 5.33 & 4.71 & 10.04 \\
\hline & VII & 7.83 & 8.99 & 16.82 & 10.18 & 10.34 & 20.52 & 1.24 & 2.73 & 3.97 & 1.36 & 3.14 & 4.50 \\
\hline & XIII & 7.17 & 4.75 & 11.92 & 7.71 & 5.46 & 13.17 & 2.59 & 3.18 & 5.77 & 1.72 & 3.29 & 5.01 \\
\hline \multirow[t]{6}{*}{ Media } & III & 1.41 & 14.25 & 15.66 & 1.84 & 16.38 & 18.22 & 9.60 & 1.49 & 11.09 & 10.26 & 1.71 & 11.97 \\
\hline & IV & 18.06 & 17.80 & 35.86 & 15.43 & 20.47 & 35.90 & 9.04 & 2.11 & 11.15 & 10.40 & 1.40 & 11.80 \\
\hline & VIII & 18.39 & 18.56 & 36.95 & 21.68 & 21.34 & 43.02 & 7.54 & 7.02 & 14.56 & 8.67 & 7.68 & 16.35 \\
\hline & IX & 6.88 & 7.35 & 14.23 & 3.07 & 7.73 & 10.80 & 3.79 & 1.09 & 4.88 & 4.36 & 1.25 & 5.61 \\
\hline & V & 7.05 & 1.85 & 8.90 & 2.50 & 0.00 & 2.50 & 0.96 & 0.42 & 1.38 & 0.39 & 0.14 & 0.53 \\
\hline & XII & 2.22 & 0.75 & 2.97 & 2.22 & 0.86 & 3.08 & 0.03 & 1.92 & 1.95 & 0.03 & 2.09 & 2.12 \\
\hline \multirow[t]{3}{*}{ Alta } & $x$ & 13.24 & 2.89 & 16.13 & 7.99 & 0.00 & 7.99 & 5.13 & 0.97 & 6.10 & 3.32 & 0.00 & 3.32 \\
\hline & $\mathrm{XI}$ & 13.54 & 2.24 & 15.78 & 14.07 & 0.00 & 14.07 & 1.61 & 0.65 & 2.26 & 0.75 & 0.00 & 0.75 \\
\hline & Nacional & 98.42 & 113.66 & 212.08 & 90.12 & 121.94 & 212.06 & 52.23 & 30.97 & 83.20 & 53.34 & 30.96 & 84.30 \\
\hline
\end{tabular}

Fuente: Datos observados: SAGARPA (2011), INEGI (2004) y CNA (2011) para producto agrícola y agua, respectivamente. Datos del modelo: este estudio.

Nota: $\mathrm{mmdp}=$ miles de millones de pesos.

total nacional de extracciones en el modelo es ligeramente mayor que las extracciones observadas ( $84.32 \mathrm{~km}^{3} /$ año y $83.20 \mathrm{~km}^{3} /$ año, respectivamente). La línea oscura sólida de la figura 5 muestra la ratio de las concesiones observadas y la recarga natural, mientras que la línea gris sólida muestra la ratio de las concesiones determinadas por el modelo en el escenario base con la recarga natural (ambas con escala en el eje vertical secundario). Como se ve, el modelo muestra un comportamiento muy cercano a los datos observados, manteniendo por tanto laasociación con el patrón de sobreexplotación de los acuíferos mostrado en las barras de la figura 5. Las ratios regionales entre las concesiones y las recargas son mayores para aquellas regiones que tienen el mayor porcentaje de acuíferos sobreexplotados sobre el total regional, sobre todo para los grupos de baja y media disponibilidad de agua. Con todo, en el escenario base son siete las regiones con sobreexplotación del acervo subterráneo o con extracciones en el límite de la recarga (I, II, VI, VII, VIII, IX y XIII), que son las mismas que muestran los datos observados. 
Figura 5. Acuíferos sobreexplotados y extracción subterránea respecto a la recarga natural: Datos observados y modelados

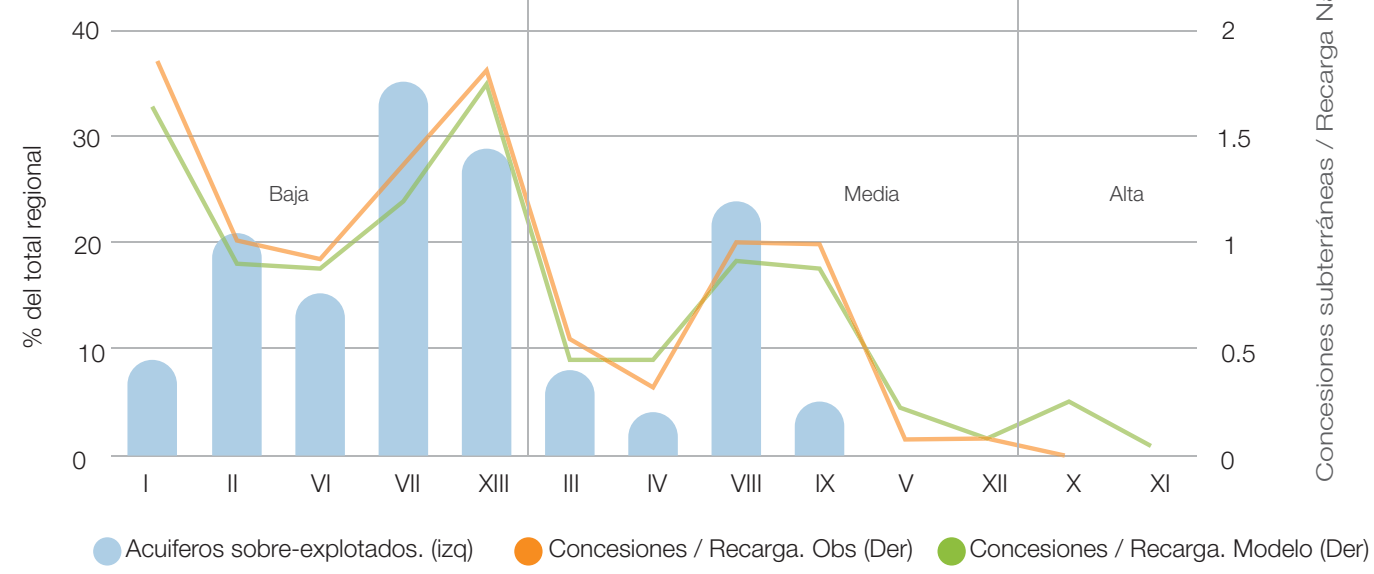

Fuente: Elaboración propia con datos de CNA (2010) y de este estudio.

5.1.1 Ventaja comparativa en la producción agrícola Los costos unitarios de producción son calculados en el modelo por la expresión

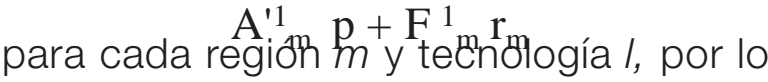
que incluyen los insumos intermedios valorados en el nivel de precios determinado por el modelo, $\mathbf{p}$, y los insumos no producidos valorados con el vector exógeno $r_{m}$. Gracias a que en el óptimo las restricciones del modelo dual se cumplen con igualdad, estos costos se pueden medir alternativamente con $\mathbf{p}-\mathbf{F}_{\mathbf{m}}^{\mathbf{l}} \mathbf{r}_{\mathbf{m}}$, es decir, con los precios unitarios de los bienes finales netos del valor unitario de los insumos escasos utilizados en su producción. La tabla 6 muestra los estructura regional de los costos unitarios de producción de los dos tipos de actividad agrícola que resultan de correr el modelo. Los costos están normalizados a los de la última tecnología activa en el modelo para generar producto agrícola, que en el escenario base es la tecnología de temporal en la región XI. Como se ve, las mayores ventajas en los costos de producción agrícola se obtienen con la agricultura de irrigación en las regiones de baja disponibilidad de agua, mientras que las regiones de alta disponibilidad de agua no exhiben ventajas comparativas en la producción de alimentos.

\subsection{Escenario de recarga} La tabla 7 muestra los resultados del escenario que limita las extracciones de agua subterránea a la recarga anual y los compara con el escenario base. En el escenario de recarga se reduce la producción de agricultura con irrigación, pues el producto nacional irrigado disminuye 5\% respecto al escenario base. Dicha disminución se localiza en cuatro regiones de baja disponibilidad de agua (I, II, VI y XIII). Para compensar por esta pérdida de producto, se requiere de una expansión de $6 \%$ en el producto agrícola de temporal, la misma que se localiza en las regiones IV, VIII y IX del grupo de disponibilidad media de agua, lo que eleva la presión sobre el uso de la tierra agrícola de temporal. Por tanto, se observa que la estrategia de sustitución de producto agrícola (de temporal por irrigado) se encuentra presente como mecanismo de ajuste ante las restricciones subterráneas. (Ver Tabla 7). 
Tabla 6. Escenario base: costos relativos unitarios de producción agrícola por región hidrológica

\begin{tabular}{lccc}
\multicolumn{3}{c}{ Disponibilidad de agua Región } & \multicolumn{2}{c}{ Costos relativos unitarios } \\
\cline { 3 - 4 } & & Temporal & Irrigación \\
\multirow{2}{*}{ Baja } & I & 0.50 & 0.21 \\
& II & 0.54 & 0.25 \\
& VI & 0.47 & 0.26 \\
& VII & 0.67 & 0.46 \\
Media & XIII & 0.87 & 0.64 \\
& III & 0.52 & 0.24 \\
& IV & 0.52 & 0.40 \\
& VIII & 0.55 & 0.40 \\
Alta & IX & 0.38 & 0.38 \\
& V & 0.87 & 0.92 \\
& XII & 0.91 & 0.83 \\
& X & 0.83 & 1.01 \\
& XI & 1.00 & 1.40 \\
\end{tabular}

Nota: Los costos están normalizados a los de la tecnología que fija el precio nacional, que en este caso es la agricultura de temporal en la región XI, en el grupo de alta disponibilidad de agua.

Fuente: Este estudio.

Al mismo tiempo, las restricciones al uso de agua subterránea produce una reducción en las extracciones de esta fuente de 3.1 $\mathrm{km}^{3} /$ año respecto al escenario base, mientras que requiere un incremento menor, de $1.1 \mathrm{~km}^{3} / a n ̃ o$, en las extracciones de agua superficial. La razón de esta correspondencia imperfecta es la posibilidad analizada en la primera estrategia: debido a que se sustituye producto irrigado con producto de temporal, no es necesario sustituir la disminución en las en la misma proporción de las superficiales. La reducción en las extracciones del acervo subterráneo se localiza en las regiones I, II, VII, XIII, del grupo de disponibilidad baja de agua, y en la región IV del grupo de disponibilidad media. Las expansiones en el uso de agua superficial también se localizan en las regiones I, II, VII, y XIII, del grupo de baja disponibilidad, resultado que se explica por la alta ventaja relativa en los costos de producción agrícola allí localizada.

Tabla 7. Escenario base y escenario de recarga: distribución regional del producto agrícola por tecnología agrícola y de las extracciones de agua tipo de fuente

\begin{tabular}{|c|c|c|c|c|c|c|c|c|c|c|c|c|c|}
\hline \multirow{3}{*}{\multicolumn{2}{|c|}{$\begin{array}{l}\text { Disponibilidad Región } \\
\text { de agua }\end{array}$}} & \multicolumn{6}{|c|}{ Producto agrícola (mmdp) } & \multicolumn{6}{|c|}{ Extracciones de agua ( $\mathrm{Km}^{3} /$ año) } \\
\hline & & \multicolumn{3}{|c|}{ Escenario Base } & \multicolumn{3}{|c|}{ Escenario Recarga } & \multicolumn{3}{|c|}{ Escenario Base } & \multicolumn{3}{|c|}{ Escenario Recarga } \\
\hline & & Tempora & Irrigación & Total & Temporal & Irrigación & Total & Superficial & Subterránea & Total & Superficial & Subterráne & Total \\
\hline \multirow[t]{5}{*}{ Baja } & 1 & 0.14 & 9.69 & 9.83 & 0.14 & 7.13 & 7.27 & 2.11 & 2.37 & 4.48 & 2.29 & 1.25 & 3.54 \\
\hline & $\|$ & 0.19 & 13.95 & 14.14 & 0.19 & 13.92 & 14.11 & 4.64 & 3.18 & 7.82 & 4.68 & 3.13 & 7.81 \\
\hline & VI & 3.10 & 15.72 & 18.82 & 3.10 & 15.72 & 18.82 & 5.33 & 4.71 & 10.04 & 4.87 & 5.17 & 10.04 \\
\hline & VII & 10.18 & 10.34 & 20.52 & 10.18 & 8.13 & 18.31 & 1.36 & 3.14 & 4.50 & 1.43 & 2.27 & 3.70 \\
\hline & XIII & 7.71 & 5.46 & 13.17 & 7.42 & 4.99 & 12.41 & 1.72 & 3.29 & 5.01 & 2.98 & 1.83 & 4.81 \\
\hline \multirow[t]{6}{*}{ Media } & III & 1.84 & 16.38 & 18.22 & 1.84 & 16.38 & 18.22 & 10.26 & 1.71 & 11.97 & 10.26 & 1.71 & 11.97 \\
\hline & IV & 15.43 & 20.47 & 35.90 & 20.19 & 20.47 & 40.66 & 10.40 & 1.40 & 11.80 & 10.40 & 1.35 & 11.75 \\
\hline & VIII & 21.68 & 21.34 & 43.02 & 21.68 & 21.34 & 43.02 & 8.67 & 7.68 & 16.35 & 8.67 & 7.68 & 16.35 \\
\hline & IX & 3.07 & 7.73 & 10.80 & 3.88 & 7.74 & 11.62 & 4.36 & 1.25 & 5.61 & 4.36 & 1.25 & 5.61 \\
\hline & v & 2.50 & 0.00 & 2.50 & 2.50 & 0.00 & 2.50 & 0.39 & 0.14 & 0.53 & 0.39 & 0.14 & 0.53 \\
\hline & XII & 2.22 & 0.86 & 3.08 & 2.22 & 0.86 & 3.08 & 0.03 & 2.09 & 2.12 & 0.03 & 2.09 & 2.12 \\
\hline \multirow[t]{3}{*}{ Alta } & $x$ & 7.99 & 0.00 & 7.99 & 7.99 & 0.00 & 7.99 & 3.32 & 0.00 & 3.32 & 3.32 & 0.00 & 3.32 \\
\hline & $X I$ & 14.07 & 0.00 & 14.07 & 14.07 & 0.00 & 14.07 & 0.75 & 0.00 & 0.75 & 0.75 & 0.00 & 0.75 \\
\hline & Nacional & 90.12 & 121.94 & 212.06 & 95.40 & 116.68 & 212.08 & 53.34 & 30.96 & 84.30 & 54.43 & 27.87 & 82.30 \\
\hline
\end{tabular}

Fuente: Este estudio.

Nota: mmdp, miles de millones de pesos 
El análisis de los resultados permite vislumbrar un tercer mecanismo de ajuste, no previsto al iniciar el ejercicio: es posible sustituir el uso de agua subterránea en algunas regiones con un mayor uso en otras. Esto sucede con las extracciones de agua subterránea en la región $\mathrm{VI}$, que pasan de 4.71 $\mathrm{km}^{3} /$ año a $5.17 \mathrm{~km}^{3} / \mathrm{año}$, de hecho ahora agotando la magnitud de la recarga natural. En el escenario de recarga, por construcción, ninguna región opera sobreexplotando los acervos subterráneos, pero siguen siendo siete regiones (I, II, VI, VII, VIII, IX, y XIII) las que operan en el límite de la recarga.

Finalmente, la tabla 8 examina el comportamiento de los precios de los bienes producidos determinados por las soluciones al modelo dual en los escenarios base y de recarga. Tradicionalmente, el modelo básico de precios basado en economía insumoproducto genera precios de los bienes producidos equivalentes a la unidad (ver, para una aplicación reciente, Perese, 2010). Sin embargo, el modelo básico de precios de Leontief no incorpora restricciones en el uso de los insumos no producidos en la forma de dotaciones finitas, a la sazón condición suficiente para la emergencia de rentas de escasez que no están contabilizadas en las transacciones de mercado representadas en las matrices insumo-producto. Por esta razón, la resolución del modelo dual con dotaciones finitas no necesariamente genera precios unitarios, sino que pueden ser mayores a la unidad. Tal es el caso de los precios de los bienes no producidos en el escenario base.

La tabla 8 informa que el escenario de recarga provoca un incremento en el precio del producto agrícola de $11 \%$ con respecto al escenario base. Este incremento se explica por la sustitución de agricultura de irrigación por agricultura de temporal, que resulta menos eficiente en cuanto a costos de producción (ver tabla 6). De igual forma, la tabla indica que si bien el incremento se concen-

Tabla 8. Precios de los bienes producidos en los escenarios base y de recarga

\begin{tabular}{|c|c|c|c|}
\hline Sector & $\begin{array}{l}\text { cios de los b } \\
\text { enario base }\end{array}$ & $\begin{array}{l}\text { ienes producidos } \\
\text { Escenario recarga }\end{array}$ & $\mathrm{Km}^{3} / \mathrm{año}$ \\
\hline Agricultura & 1.20 & 1.34 & 1.11 \\
\hline Ganadería & 1.77 & 1.80 & 1.02 \\
\hline Petróleo y gas & 1.15 & 1.15 & 1.01 \\
\hline Minería & 1.52 & 1.52 & 1.00 \\
\hline Electricidad & 1.60 & 1.62 & 1.01 \\
\hline Construcción & 1.29 & 1.32 & 1.02 \\
\hline Alimentos procesados & 1.24 & 1.27 & 1.03 \\
\hline Manufactura ligera & 1.22 & 1.25 & 1.02 \\
\hline Plásticos y químicos & 1.23 & 1.25 & 1.01 \\
\hline Manufactura pesada & 1.19 & 1.21 & 1.02 \\
\hline Comercio & 1.27 & 1.31 & 1.04 \\
\hline Transporte & 1.48 & 1.54 & 1.04 \\
\hline Servicios no financieros & 1.42 & 1.46 & 1.02 \\
\hline Servicios financieros & 1.46 & 1.49 & 1.02 \\
\hline Otros servicios & 1.34 & 1.40 & 1.04 \\
\hline
\end{tabular}

Fuente: Este estudio. 
tra en el precio agrícola, existe un efecto de contagio, aunque moderado, en el precio del resto de los sectores económicos. La magnitud de este contagio será mayor en sectores que mantengan mayores vínculos con el sector agrícola (por ejemplo, sectores de comercio y de transporte). Como es normal en la economía insumo-producto, las magnitudes mostradas en la tabla 9 representan los efectos totales, directos e indirectos, de imponer restricciones a las concesiones de uso de agua subterránea.

\section{Conclusión}

En este documento se presenta un modelo de comercio interregional aplicado para el análisis de usos y disponibilidad de agua en México. Aplicaciones previas del mismo incluyen el tratamiento diferenciado del sector agrícola por tipo de tecnología (temporal o de irrigación) y distinguen las dotaciones de agua en el nivel regional utilizando las 13 regiones hidrológicoadministrativas en las que la CNA mexicana divide al territorio. En este documento se expande dicho tratamiento distinguiendo dos fuentes de agua renovable, superficial y subterránea, para estudiar la dependencia de la actividad económica sobre cada una. En particular, este estudio se enfocó en el examen de la sobreexplotación de los acervos subterráneos de agua y se estudian los mecanismos de ajuste económico ante la implementación de restricciones a la explotación de los acuíferos.

A partir de una calibración para datos del año 2004, que corresponde a la última tabla insumo-producto publicada oficialmente, se formularon dos escenarios con el modelo referido. Un escenario base sirve como referencia para la comparación con los resultados de un escenario que restringe las extracciones de agua subterránea a la recarga media anual regional. El análisis de escenarios permitió identificar tres mecanismos de ajuste ante tales restricciones. Primero, la sustitución de agricultura de irrigación por agricultura de temporal, que no implica presiones sobre los acervos de agua aquí tratados. Segundo, la sustitución de fuentes superficiales por fuentes subterráneas. Y tercero, la sustitución de extracción subterránea en algunas regiones por expansiones de la extracción subterránea en otras regiones.

Los resultados informan que el primer mecanismo de ajuste es el dominante. En el escenario que limita las extracciones subterráneas a la recarga, el producto agrícola de irrigación disminuye en 5\%, principalmente en las regiones de disponibilidad baja de agua y en las que se observa la mayor sobreexplotación de los acuíferos, y es compensado por una expansión de $6 \%$ en el producto de agricultura de temporal, localizada en las regiones de disponibilidad media de agua. El segundo mecanismo de ajuste también se encuentra presente, pero en menor medida: en el escenario de recarga las extracciones subterráneas caen en $3 \mathrm{~km}^{3} /$ año y las superficiales aumentan en $1 \mathrm{~km}^{3}$ / año. La ausencia de proporcionalidad en esta sustitución se posibilita por el dominio del primer mecanismo de ajuste, en el que la expansión de agricultura de temporal compensa la caída en la agricultura de irrigación.

En el tercer mecanismo de ajuste, que no estaba no previsto en el diseño del experimento aquí reportado, una menor extracción subterránea en las regiones de baja disponibilidad obliga a una expansión de las mismas en la región VI del grupo de disponibilidad media. Dicha expansión, sin embargo, es menor que las disminuciones requeridas, de modo tal que el efecto neto nacional en las extracciones subterráneas es negativo, como queda dicho arriba. 
El modelo aquí presentado es un marco de análisis que puede ser utilizado para estudiar los mecanismos económicos de ajuste ante escenarios de escasez y de sustentabilidad en el uso del agua. Las extensiones posibles incluyen un tratamiento más fino del sector agrícola con el fin de distinguir por rama de producto (por ejemplo, vegetales, granos, otros) y representar con mayor detalle su dependencia a las tecnologías agrícolas de riego y temporal. También es posible ampliar el análisis de la sustentabilidad al uso del agua superficial, que implica tomar en cuenta los requerimientos de agua de los ecosistemas. Por último, cabe realizar escenarios que levanten el supuesto de un comercio constante con el resto del mundo, implícito en este tratamiento, con el fin de incluir modificaciones a las exportaciones netas como otro de los mecanismos de ajuste ante las condiciones de sustentabilidad del uso del agua.
AGRADECIMIENTOS El autor agradece los comentarios de dos revisores anónimos, muchos de los cuales han sido incorporados. Cualquier error que pueda aparecer es, por supuesto, plena responsabilidad del autor. 


\section{REFERENCIAS BIBLIOGRÁFICAS}

Berrittella, M., K. Rehdanz, R. Roson, \& R Tol (2005) "The economic impact of water pricing: A computable general equilibrium analysis", en Working Paper FNU-96, Universidad de Hamburgo y Centro para la Ciencia Marina y Atmosférica, Hamburgo, Alemania.

Berrittella, M., A. Hoekstra, K. Rehdanz, R. Roson, \& R. Tol (2007) "The economic impact of restricted water supply: A computable general equilibrium analysis", en Water research, 41(8):1799-1813.

Blancas, A. (2006) "Interinstitutional linkage analysis: a social accounting matrix multiplier approach for the Mexican economy", en Economic Systems Research, 18(1):29-59.

Calzadilla, A., K. Rehdanz \& R. Tol (2008) "Water scarcity and the impact of improved irrigation management: a CGE analysis", en Kiel Working Papers No.1436, Instituto Kiel para la Economía Mundial, Kiel, Alemania.

CNA (2010) Estadísticas del Agua en México 2010. Secretaría del Medio Ambiente y Recursos Naturales. México D.F.

Duarte, R., J. Sánchez-Chóliz, \& J. Bielsa (2002) "Water use in the Spanish economy: an input-output approach", en Ecological Economics, 43(1):71-85.

Duchin, F. (2005) "A world trade model based on comparative advantage with $m$ regions, $n$ goods, and k factors", en Economic Systems Research, 17(2):141-162

Duchin, F. \& C. López-Morales (2011) “World trade in food as an adjustment mechanism to water scarcity", en Conferencia 19 de la Sociedad Internacional Insumo-Producto, Alexandria, Virginia, EUA. En prensa.
Ehrlich, P., E. Anne \& J. Holdren (1977) Ecoscience: population, resources, environment. San Francisco. WH Freeman and Comp.

FAO (2009) Aquastat Information System. Base de datos electrónica. Consultado: 7 de mayo de 2012. http://www.fao.org/nr/ water/aquastat/main/index.stm

Gleick, P. (2009) The World's Water 20082009: The Biennial Report on Freshwater Resources. Washington, D.C, Island Press

Guan, D. \& K. Hubacek (2008) "A new and integrated hydro-economic accounting and analytical framework for water resources: A case study for North China", en Journal of Environmental Management, 88(4):13001313.

Harris, T. \& M. Rea (1984) "Estimating the value of water among regional economic sectors using the 1972 national inter-industry format", en American Water Resources Association, 20(2):193-201.

He, J., X. Chen \& Yong Shi (2006) "A dynamic approach to calculate shadow prices of water resources for nine major rivers in China", en Journal of Systems Science and Complexity, 19(1):76-87.

He, J., X. Chen, Y. Shi \& A. Li (2007) "Dynamic computable general equilibrium model and sensitivity analysis for shadow price of water resource in China", en Water Resources Management, 21(9):1517-1533.

He, L. \& y F. Duchin (2009) "Regional development in China: Interregional transportation infrastructure and regional comparative advantage", en Economic Systems Research, 21(1):3-22. 
Henry, M. \& E. Bowen (1981) "A method for estimating the value of water among sectors of a regional economy", en Southern Journal of Agricultural Economics, 13(2):125-132.

Hubacek, K. \& L. Sun (2005) "Economic and societal changes in China and their effects on water use", en Journal of Industrial Ecology, 1(2):187-200.

ICWE (1992) The Dublin Statement and Report of the Conference, International Conference on Water and the Environment: Development Issues for the 21st century, Dublin.

INEGI (2012). Sistema de consulta de los censos económicos 2004. Base de datos electrónica. [Página web] Consultado: 7 de mayo de 2012. http://www.inegi.org.mx

INEGI (2012a). Sistema de Cuentas Nacionales: Matriz de Insumo Producto. Base de datos electrónica. [Página web] Consultado: 7 de mayo de 2012. http://www.inegi.org. mx/est/contenidos/Proyectos/SCN/C_ Anuales/matrizinsumo/default.aspx

Juliá, R. \& F. Duchin (2007) "World trade as the adjustment mechanism of agriculture to climate change", en Climatic Change, 82(3):393-409 .

Lee Harris, R. (2002) "Estimation of a regionalized Mexican Social Accounting Matrix using entropy techniques to reconcile disparate data sources", en Trade and Macroeconomic Division Discussion Papers No. 97, International Food Policy Research Institute, Washington, D.C.

Lenzen, M. (2009) "Understanding virtual water flows: A multiregion inputoutput case study of Victoria", en Water Resources Research, 45, W09416, doi:10.1029/2008WR007649.

Lenzen, M. \& B. Foran (2001) "An Input-Out- put Analysis of Australian Water Usage", en Water Policy, 3, 321-340.

Liu, X. X. Chen, \& S. Wang (2009) "Evaluating and predicting shadow prices of water resources in China and its nine major river basins", en Water Resources Management, 23(8):1467-1478

Llop, M. (2008) "Economic impact of alternative water policy scenarios in the Spanish production system: An input-output analysis", en Ecological Economics, 68(1-2):288294.

López-Morales, C. \& F. Duchin (2011) "Policies and Technologies for a Sustainable Use of Water in Mexico. A Scenario Analysis", en Economic Systems Research 23(4): 387407.

Perese, K. (2010) "Input-Output Model Analysis: Pricing Carbon Dioxide Emissions", en Working Papers Series 2010-4, Congressional Budget Office, Washington, D.C.

Postel, S., G. Daily \& P. Ehrlich (1996) "Human appropriation of renewable fresh water", en Science, 271(5250):785

SAGARPA (2009). Sistema de Información Agropecuaria y Pesquera. Anuarios Agropecuarios 1980-2008. Base de datos electrónica. [Página web] Consultado: 2 de diciembre de 2011.

http://www.campomexicano.gob.mx/portal_siap/PublicaDinamica/SisInformacion/ Anuario_2008/anuario_1980-2008_wv.html

Seckler, D. (1996) "The New Era of Water Resources Management: From "Dry" to "Wet" Water Savings", en Research Report 1, International Water Management Institute, Colombo.

SEMARNAT (2009) Sistema Nacional de Información Ambiental y Recursos Natura- 
les. Base de datos electrónica. [Página web] Consultado: 1 de diciembre de 2011. http://www.semarnat.gob.mx/informacionambiental/Pages/sniarn.aspx

Strömman, A. \& F. Duchin (2006) "A world trade model with bilateral trade based on comparative advantage", en Economic Systems Research, 18(3):281-297.

Strömman, A., E. Hertwich \& F. Duchin (2009) "Shifting trade patterns as a means of reducing global carbon dioxide emissions: A Mul- tiobjective Analysis", en Journal of Industrial Ecology, 13(1):38-57

Velázquez, E. (2006) "An input-output model of water consumption: Analysing intersectoral water relationships in Andalusia", en Ecological Economics, 56(2):226-240. 